\title{
The erosion of physical activity in Western societies: an economic death march
}

\author{
Frank W. Booth ${ }^{1,2}$ • John A. Hawley ${ }^{3,4}$
}

Received: 20 February 2015 / Accepted: 18 March 2015 / Published online: 8 May 2015

(C) Springer-Verlag Berlin Heidelberg 2015

\begin{abstract}
By 2030 type 2 diabetes and associated complications will be the seventh leading cause of death globally. In this context, obesity and physical inactivity have emerged as major risk factors for several chronic metabolic disorders. While exercise training exerts numerous health-related benefits in terms of the prevention and treatment of many disease states, including type 2 diabetes, it is currently underprescribed and under-valued. We contend that unless urgent action is taken to curb the tidal wave of inactivity-related metabolic diseases, the worldwide economic burden associated with the rise in the number of diagnosed cases of type 2 diabetes will trigger the start of an economic death march for both industrial and developing nations alike. In this commentary we look ahead to 2065 and consider the impact that lifestyle interventions and associated strategies are likely to have in curbing the epidemic tide of type 2 diabetes. This is one of a series of commentaries under the banner ' 50 years forward', giving personal opinions on future perspectives in diabetes, to celebrate the 50th anniversary of Diabetologia (1965-2015).
\end{abstract}

John A. Hawley

john.hawley@acu.edu.au

1 Department of Biomedical Sciences, University of Missouri, Columbia, MI, USA

2 Department of Nutrition and Exercise Physiology, University of Missouri, Columbia, MI, USA

3 Center for Exercise and Nutrition, MacKillop Institute for Health Research, Australian Catholic University, Locked Bag 4115, Fitzroy MDC, Melbourne, VIC 3065, Australia

4 Research Institute for Sport and Exercise Sciences, Liverpool John Moores University, Liverpool, UK
Keywords Exercise - Exercise mimetics - Exercise training · Obesity · Physical inactivity

\begin{abstract}
Abbreviations
HIT High-intensity interval training

IGT Impaired glucose tolerance
\end{abstract}

\section{Introduction}

There is a growing global health burden arising from the interrelated sequelae of metabolic disorders comprising impaired glucose tolerance (IGT), obesity and type 2 diabetes. Obesity and inactivity are the main drivers of IGT and type 2 diabetes and are responsible for up to $8 \%$ of health costs and $13 \%$ of deaths ( 1.5 million in 2014) worldwide, with the risk of comorbidities rising in parallel with increasing body weight [1]. The problem begins in childhood and while the trend in the incidence of overweight children may have transiently plateaued in several countries, Olds et al [2] caution that it is uncertain 'whether it is a temporary lull, and overweight and obesity rates will soon begin to rise again'. The WHO predicts that by 2030 diabetes will be the seventh leading cause of death globally [1]. While physical inactivity has emerged as a major risk factor for many chronic metabolic disorders, it is well accepted that exercise training has positive effects in terms of the prevention and treatment of almost all lifestylerelated disease states, including type 2 diabetes [3]. Indeed, there are no interventions with a higher therapeutic index than either physical exercise and/or diet manipulation (e.g. energy restriction) to reduce the risk of virtually all chronic diseases simultaneously, with little or no adverse side effects. If action is not taken soon, the worldwide economic burden associated with the rise in the number of diagnosed cases of type 2 
diabetes will trigger the start of an economic death march for both industrial and developing nations alike. Here we look forward to 2065 and contemplate the impact lifestyle interventions are likely to have in curbing the epidemic tide of type 2 diabetes.

\section{Looking to 2065 and beyond with limited optimism}

Innovative methods will improve exercise participation Part of the reason for the apathy towards modifying lifestyle habits is that current public health recommendations may be unrealistic and unattainable for the majority of the populace. Looking to 2065 and beyond, there is an urgent need for innovations in the delivery of exercise prescription so that it can be incorporated into daily living to induce clinically beneficial health outcomes for all adults. In this regard, a growing body of evidence demonstrates that low-volume, highintensity interval training (HIT) confers health benefits comparable to those afforded by traditional endurance-based training in both healthy individuals and diseased populations, with a markedly reduced exercise volume and a lower time commitment [4]. Recent work in individuals with IGT shows that 'exercise snacking' (brief bouts of HIT walking before daily meals, $18 \mathrm{~min}$ of exercise/day) results in markedly superior blood glucose control than a single bout of 'traditional' continuous exercise [5]. These findings are important from a public health perspective given that a 'lack of time' remains the most commonly cited barrier to regular exercise participation, independent of sex, age and socioeconomic status.

Technological developments will assist individuals to become more active We predict that technological developments in the next 50 years (e.g. smartphone applications with internet connection and the ability to monitor adherence to exercise sessions and food intake on a daily basis, real-time monitoring of glucose and insulin concentrations) will provide positive feedback to assist many people to become more active and become partners in medical research.

An integrative approach, including exercise, will be used to study metabolic diseases Future research in the field of exercise biology requires increasingly sophisticated approaches from various model systems to understand the critical nodes of energy homeostasis and how these pathways are disrupted in a number of chronic inactivity-related metabolic disorders [6]. Advances in genomics, epigenetics, proteomics and metabolomics in the next 50 years should present unlimited opportunities for innovative, interdisciplinary research to identify unique molecular signatures predictive of individual susceptibility to metabolic disease states and, more importantly, revolutionise personalised/precision medicine in both prevention and therapeutics (e.g. exercise genomics). In the final analyses, when interfaced with computational tools for analysing large datasets, the various 'omics' technologies will produce readouts that have translational value to whole body physiology and pathology.

Exercise mechanisms will be determined to improve healthspan and thus compress mortality Fries described the 'ideal survivorship curve' hypothesis, in which he envisioned a potential delay of the onset of the first chronic disease/disorder and of healthcare costs nearer in time to the age of death [7]. Today, the terms 'lengthening of healthspan' and 'compression of morbidity' are used to describe Fries' ideal curve. We envisage that residents in assisted-living facilities will have daily 'gym' periods run by exercise physiologists and physical therapists. In 2065, physically active lifestyles will have compressed the period of morbidity into the last years or even months of life.

\section{Looking to 2065 and beyond with pessimism}

The global increase in the prevalence of type $\mathbf{2}$ diabetes will not abate The above prognosis for the next 50 years was written from the perspective of an optimistic agenda. However, the facts do not support our optimism (Fig. 1). One important statistic suggesting a pessimistic future is the projection for an increased prevalence of type 2 diabetes. According to projections by the International Diabetes Federation, by 2030 more than 138 million Europeans will have either IGT or overt type 2 diabetes [8], while the US Centers for Disease Control and Prevention (CDC) predicts that if current trends continue, one out of three adults in the USA will have type 2 diabetes by 2050 [9]. Controversy exists regarding the direct healthcare costs of diabetes in various countries, but they range from $2.5 \%$ to $15 \%$ of annual healthcare budgets, depending on local diabetes prevalence and the sophistication of the treatments available. While direct costs for healthcare expenditure are quantifiable, they fail to consider indirect costs to the individual and family, such as pain and suffering, anxiety, a lower quality of life, reduced mobility, loss of earnings and premature retirement. The facts regarding IGT and type 2 diabetes imply that the more likely outcome in 50 years is that more humans will be sicker and die sooner because of a lack of daily physical activity.

Exercise mimetics will not replace all of the benefits obtained from exercise training Recognising the combined benefits of exercise (both endurance- and resistance-based training) in the prevention and treatment of many chronic metabolic diseases, along with the trend towards increasing inactivity at the population level, there has recently been a 


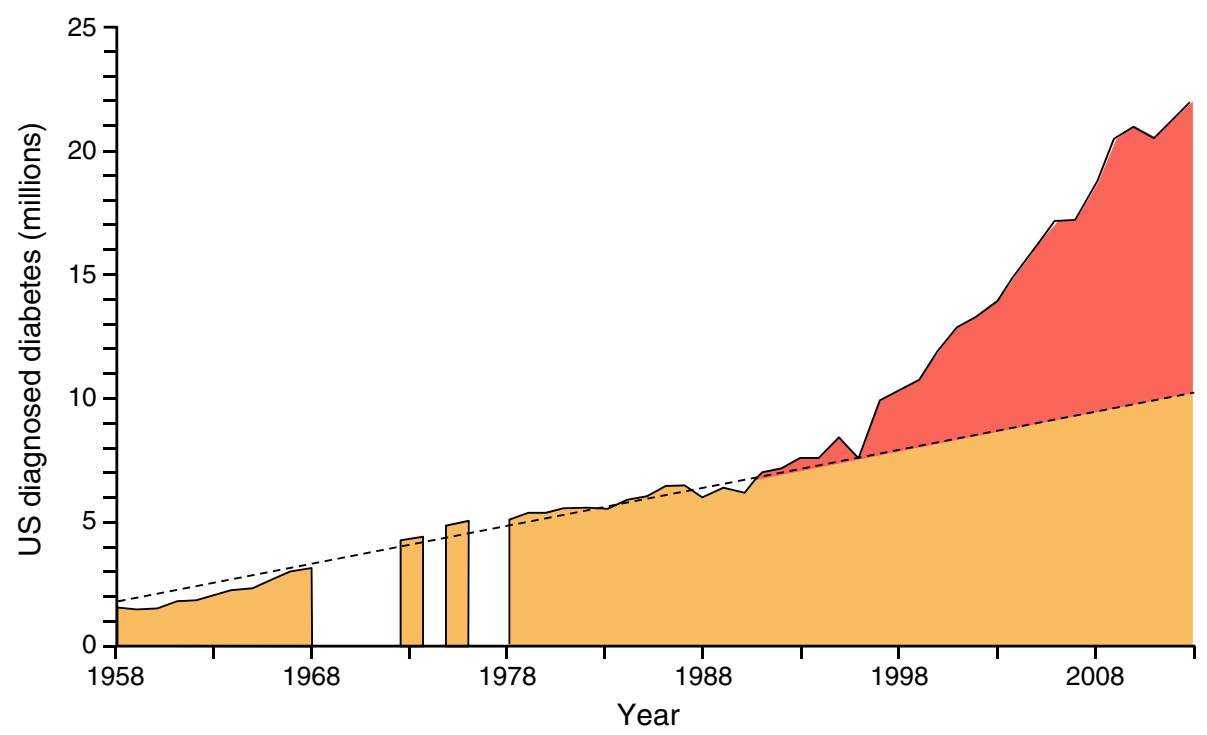

Fig. 1 Increase in diagnosed diabetes the USA during the 56 year period (1958-2013; with 6 years without data collection) [9]. The increase is divided into two parts to suggest at least two potential causes. A warningamber area is enclosed below a dashed straight line to suggest one set of causes from 1958-2013. During the 1990s, another set of causes initiates

large-scale commercial effort to discover orally active compounds that potentiate some minimal effects of exercise, the so-called 'exercise mimetics'. Although it is possible to mimic a 'small' part of an exercise effect by exposing skeletal muscles to chemical agents that activate transcription factors with putative roles in training-induced adaptation (i.e. the AMPactivated protein kinase and the peroxisome proliferatoractivated receptor coactivators), the complexity of exercise as a multi-organ/multi-cell type stimulus makes it highly unlikely that any single pharmacological approach could ever mimic such wide-ranging effects [6]. While a 'polypill' containing several compounds capable of activating tissue-specific, exercise-induced targets is a remote possibility, the likelihood of multiple off-target and potential deleterious side effects would likely outweigh any health-conferring benefit, preventing legalisation of the drug. We contend that the term 'exercise mimetic' is being used somewhat unethically, because the pharmacological examples of its purported benefits fail to mimic the majority of the health-related benefits of actual exercise. Finding ways to motivate people to adopt and maintain a physically active lifestyle is likely to have a greater impact on individual and population health than searching for potential pharmacological treatments [6].

\section{Precision medicine initiative fails to include mention of} physical activity modifications The Human Genome project was supposed to pave the way for personalised therapeutic and preventive medicine, with 'genetic readouts' making it possible for clinicians to predict individuals at high risk for many common diseases and their responsiveness to specific treatment strategies. Twenty years ago it was believed an exponential increase above the dashed line, the area of which is shaded red to signal a need to stop the increase. Our current commentary contends that a physically inactive lifestyle is one factor that contributes to both the amber and red areas

(somewhat naively for complex diseases and lifestyles) that a few selected genetic variants (single nucleotide polymorphisms, or SNPs) would explain much of an individuals' inherited risk for several common diseases. However, lifestyle-related disorders like type 2 diabetes are progressive metabolic diseases that are polygenic in nature and develop from both environmental and too many genetic factors, each of which make too small a percentage contribution to be useful clinically. The complete absence of the words 'exercise' and 'physical activity' as potent primary measures to combat lifestyle-related chronic diseases in the Precision Medicine Initiative [10], foretells that the US government at least has left the future of its citizens' health to the fate of biological destiny. The continued search for 'personalised/precision' medicine to improve care and speed the development of new treatments will, we believe, provide less than the achievable optimal health.

Medical school curriculums will continue to overemphasise secondary rather than primary preventative strategies to combat lifestyle-related metabolic diseases As part of the wider strategy to increase society's physical activity levels, education of healthcare professionals is a key element in the delivery of appropriate primary preventative strategies to individuals in local communities. One of the principal 'gatekeepers' in the provision of healthcare delivery, and often the initial point of contact when an individual seeks medical advice, is the role of general practitioners. Yet, due to a lack of appropriate training in the fields of exercise physiology/exercise prescription and nutrition, the vast majority of general practitioners are ill-equipped to 
impart the latest information regarding exercise and/or nutrition interventions or long-term lifestyle modifications. In support of this contention, a recent study revealed major limitations in knowledge of physical activity guidelines among UK medical students, who underestimated the risk of physical inactivity and did not know the physical activity guidelines as well as other health promotion guidelines [11]. Moreover, a large proportion remained unconfident about giving advice regarding exercise prescription [11]. Clearly, if we are to attack the root of the problem underlying many chronic inactivity-related metabolic diseases, such as type 2 diabetes, then improved education of healthcare providers is urgently required.

\section{Suboptimal physical development of children and adoles-} cents from insufficient levels of physical activity will continue In the USA, $42 \%$ and $92 \%$ of $6-11$-year-olds and 12-19-year-olds, respectively, do not meet the recommendations of 60 min of daily physical activity [12]. As a result, it is likely that many children do not reach their optimal physical capacity. In the third decade of life, children will have compromised aerobic capacities, along with suboptimal skeletal muscle mass. The long-term clinical consequences will be that children will be at higher risk of prematurely developing chronic diseases associated with adulthood, such as type 2 diabetes. Prior to 1990, type 2 diabetes was rare in people under 40 years old. Two decades later, type 2 diabetes in children and adolescents is becoming an increasingly important public health concern throughout the world [13]. Furthermore, physically undeveloped children/adolescents will reach physical (sarcopenia) and aerobic (cardiorespiratory) fragility at an earlier age. The last decade of a shortened lifespan will be inactive, with the individual living in assisted care. Sarcopenia is exacerbated in the overweight/obese person, promoting an increased fat mass and a concomitant decrease in muscle mass [14]. Sarcopenia not only reduces mobility and functional capacity, but also accelerates obesity and progression towards type 2 diabetes in a spiral of 'cumulative causation' [14].

\section{Summary: our pessimisms are based upon a history of exercise science falling on deaf ears}

Figure 1 illustrates a prediction made by the US government. In 2050, 149 million US residents have been predicted to have type 2 diabetes in their lifetime. To put these numbers in perspective, there were 1.6 million US cases of type 2 diabetes in 1960, which means that the prediction represents a 93-fold increase! The WHO forecasts that in some parts of the world there will be an even higher future prevalence of type 2 diabetes than predicted for the USA [1]. We seem content to condemn a large proportion of future generations to a life of chronic metabolic disease that will only bring unbearable personal suffering and accelerate a global economic death march.

Some exercise scientists have shown a willingness to tackle the unjustness of our children having unacceptably high odds of acquiring the preventable lifestyle disease of type 2 diabetes. However, past efforts have been ineffective in convincing both scientific and political leaders that physical activity is crucial to the prevention of type 2 diabetes and must begin with the prescription and facilitation of childhood activity. Exercise scientists have been largely ineffective in convincing those in power to act to stop the rise in type 2 diabetes. One potential barrier perceived by the authors is that exercise scientists are considered to be doing less than 'high calibre' science by other fields of medical sciences. Thus, many exercise scientists have been unable to climb the 'scientific ladder' deemed necessary to voice their concerns and convince political leaders (the majority of whom are not trained in the health sciences) of the value of primary disease prevention through exercise participation. To increase awareness of the impending economic death march to type 2 diabetes, we need to take new directions to reach the ears of those political leaders who are willing to help. We contend that we all need to demand that society stops harming vulnerable children with a genetic predisposition to type 2 diabetes and other inactivity-related metabolic disorders so that they can live healthy lives: parents should not be allowed to prevent their children's future freedom from type 2 diabetes. Such a contention is not novel. Many free societies outlaw tobacco use for minors. Most governments do not allow children to vote. Political leaders need to facilitate opportunities for a physically active lifestyle. For example, they need to offer incentives, such as tax credits to parents whose children meet physical activity guidelines/thresholds. Such monetary incentives already exist in some US private companies in the form of healthcare savings plans. Getting paid to be healthy is less costly than getting paid to be sick! There is no time to lose, as our children are growing older and, in many cases, unhealthier with every passing day.

Acknowledgements The authors would like to thank R. Toedebusch, G. Ruegsegger (both University of Missouri, Columbia, MO, USA) and C. Roberts (Pritikin Research Foundation, Los Angeles, CA, USA) for conceptual ideas in the preparation of this article.

Duality of interest The authors declare that there is no duality of interest associated with this manuscript.

Contribution statement FWB and JAH were both responsible for the conception of this manuscript, drafting the article and approving the final version for publication. 


\section{References}

1. World Health Organisation Diabetes Fact Sheet \#32 (2015) Available from www.who.int/mediacentre/factsheets/fs312/en/, accessed Jan 2015

2. Olds T, Maher C, Zumin S et al (2011) Evidence that the prevalence of childhood overweight is plateauing: data from nine countries. Int J Pediatr Obes 6:342-360

3. Booth FW, Gordon SE, Carlson CJ, Hamilton MT (2000) Waging war on modern chronic diseases: primary prevention through exercise biology. J Appl Physiol 88:774-787

4. Gibala MJ, Little JP, Macdonald MJ, Hawley JA (2012) Physiological adaptations to low-volume, high-intensity interval training in health and disease. J Physiol 590:1077-1084

5. Francois ME, Baldi JC, Manning PJ et al (2014) 'Exercise snacks' before meals: a novel strategy to improve glycaemic control in individuals with insulin resistance. Diabetologia 57:1437-1445

6. Hawley JA, Hargreaves M, Joyner MJ, Zierath JR (2014) Integrative biology of exercise. Cell 159:738-749

7. Fries JF (1980) Aging, natural death, and the compression of morbidity. N Engl J Med 303:130-135
8. International Diabetes Federation (2014) IDF Diabetes Atlas: Europe (Sixth Edition). Available from www.idf.org/diabetesatlas/ europe, accessed Apr 2015

9. Long-term Trends in Diabetes (2014) CDC's Division of Diabetes Translation. National Diabetes Surveillance System. Available from www.cdc.gov/diabetes/statistics/slides/long_term_trends.pdf, accessed Apr 2015

10. Collins FS, Varmus H (2015) A new initiative on precision medicine. N Engl J Med 372:793-795

11. Dunlop M, Murray AD (2013) Major limitations in knowledge of physical activity guidelines among UK medical students revealed: implications for the undergraduate medical curriculum. Br J Sports Med 47:718-720

12. Troiano RP, Berrigan D, Dodd KW, Mâsse LC, Tilert T, McDowell M (2008) Physical activity in the United States measured by accelerometer. Med Sci Sports Exerc 40:181-188

13. Rosenbloom AL, Silverstein JH, Amemiya S, Zeitler P, Klingensmith GJ (2009) Type 2 diabetes in children and adolescents. Pediatr Diabetes 10(Suppl 12):S17-S32

14. Parr EB, Coffey VG, Hawley JA (2013) 'Sarcobesity': a metabolic conundrum. Maturitas 74:109-113 